\title{
Autism and ADHD Symptoms in Patients with OCD: Are They Associated with Specific OC Symptom Dimensions or OC Symptom Severity?
}

\author{
Gideon E. Anholt • Danielle C. Cath • Patricia van Oppen • \\ Merijn Eikelenboom • Johannes H. Smit • Harold van Megen • \\ Anton J. L. M. van Balkom
}

Published online: 29 December 2009

(C) The Author(s) 2009. This article is published with open access at Springerlink.com

\begin{abstract}
In obsessive-compulsive disorder (OCD), the relationship between autism spectrum disorders (ASD), attention-deficit/hyperactivity disorder (ADHD) symptom, and obsessive-compulsive (OC) symptom dimensions and severity has scarcely been studied. Therefore, 109 adult outpatients with primary OCD were compared to 87 healthy controls on OC, ADHD and ASD symptoms. OCD patients showed increased ADHD and autism symptom frequencies, OCD + ADHD patients reporting more autism symptoms (particularly attention switching and social skills problems) than OCD - ADHD patients. Attention switching problems were most significant predictors of OC symptom dimensions (except hoarding) and of symptom severity. Hoarding was not associated with elevated autism scale scores, but with inattention. In conclusion, attention
\end{abstract}

G. E. Anholt · P. van Oppen - M. Eikelenboom ·

J. H. Smit - A. J. L. M. van Balkom

Department of Psychiatry and Institute for Extramural Medicine,

VU-University Medical Centre, Amsterdam, The Netherlands

D. C. Cath · P. van Oppen - A. J. L. M. van Balkom Outpatient Clinic for Anxiety Disorders, GGZ-inGeest, Amsterdam, The Netherlands

D. C. Cath

Department of Clinical and Health Psychology, Utrecht University/Altrecht, Anxiety Outpatient Program, Utrecht,

The Netherlands

H. van Megen

Meerkanten Psychiatric Institute, Ermelo, The Netherlands

D. C. Cath $(\bowtie)$

Altrecht Anxiety Outpatient Program, Mimosastraat 2-4,

3551 DC Utrecht, The Netherlands

e-mail: cath@xs4all.nl; d.cath@uu.nl switching problems may reflect both symptom overlap and a common etiological factor underlying ASD, ADHD and OCD.

Keywords Obsessive-compulsive disorder . Attention deficit/hyperactivity disorder · Autism

\section{Introduction}

Once considered a rare phenomenon, epidemiological studies have found obsessive-compulsive disorder (OCD) to be the fourth most common psychiatric disorder (Myers et al. 1984), with prevalence rates between 1.5 and $3 \%$ in the general population (Bebbington 1998; Stein et al. 1997).

Obsessive-compulsive disorder (OCD) encompasses a relatively frequent anxiety disorder, characterized by repetitive thoughts, impulses, or images (obsessions), and repetitive behaviors or mental acts (compulsions) that cause marked distress (APA 1994). OCD is highly debilitating, and the World Health Organization has found it to be among the 10 most disabling medical conditions (Angst et al. 2004; Weissman et al. 1994; Murray and Lopez 1996). Hollander and colleagues have suggested that OCD is part of the "obsessive compulsive spectrum disorders" (Hollander and Wong 1995; Hollander et al. 1996). Disorders belonging to this spectrum are thought to share similarities in clinical symptoms, associated features, etiology, and response to treatment. The obsessive-compulsive spectrum disorders include neuropsychiatric conditions such as: Tourette's syndrome, Sydenham's chorea, and autism.

Most of the research into the relationship between autism and OCD has been performed in pediatric patients with autism spectrum disorders (ASD). Children with ASD have 
shown increased rates of obsessive-compulsive (OC) symptoms (Leyfer et al. 2006). Reversely, pediatric OCD patients show increased frequencies of autism symptoms (6.2\%; Ivarsson and Melin 2008). Considering the frequency of adults with an early onset form of OCD (about 50\%; Maina et al. 2008), it is likely that this association with ASD also holds for adults with OCD. Co-morbid tics (especially in girls with OCD) and pathological doubt seems to be the strongest predictor of autism symptoms in pediatric OCD (Ivarsson and Melin 2008). In adult OCD patients, up to $20 \%$ of the patients exhibited autistic traits (Bejerot et al. 2001). However, much less research has been carried out on symptom profiles of ASD in adults with OCD. This might partly be due to a lack of familiarity of adult clinicians with childhood-onset disorders with the consequence that ASD symptom are often missed (Cath et al. 2008). Nonetheless, knowledge about the nature of the co-morbidity between OCD and both ASD and ADHD is of great importance to the adult clinician, since it might explain non compliance to treatment and treatment resistance. Repetitive behaviors are among the core features of both ASD and OCD, and comparison of ASD with OCD has demonstrated more similarities than differences in OC symptom characteristics between these groups. Some difference can be noted: ASD patients demonstrate more hoarding, touching, tapping and self-damaging behaviors, less checking, repeating and counting behavior, and less aggressive and somatic obsessions than OCD patients (McDougle et al. 1995; Russell et al. 2005). Interestingly, adult patients with comorbid ASD and OCD report more sexual obsessions than patients with one of these disorders alone (Zandt et al. 2007; Russell et al. 2005; Cath et al. 2008). Besides symptom overlap, similar executive function impairments related to obsessions and compulsions have been found in patients and first degree relatives in both disorders (Delorme et al. 2007). Based on this overlap, Bejerot (2007) has proposed a new OCD subtype, with an autistic dimension characterized by either paranoid, schizoid, schizotypal, avoidant or obsessive-compulsive traits.

Attention-deficit/hyperactivity disorder (ADHD) is not included in the obsessive-compulsive spectrum theory. However, Palumbo and colleagues (Palumbo et al. 1997) have suggested that ADHD, OCD and autism share etiological overlap, constituting a group of developmental basal ganglia disorders. Elevated rates of ADHD symptoms have been found in OCD, with percentages up to $30 \%$ of comorbid ADHD in children and adolescents (Hanna 1995; March and Leonard 1996). Reversely, percentages of OCD in children with ADHD are estimated at $8 \%$ (Geller et al. 2000).

According to DSM IV criteria, when ADHD symptoms exclusively occur in the course of pervasive developmental disorders (PDD), a co-morbid diagnosis of ADHD is not allowed. Yet, recent clinical as well as epidemiological studies indicate that ADHD symptoms are present in up to $75 \%$ of children with PDD (Goldstein and Schwebach 2004; Sturm et al. 2004; Yoshida and Uchiyama 2004). Reversely, characteristics of ASD (such as difficulties in social interactions, and problems in verbal and nonverbal communication) have been found in children with ADHD (Clark et al. 1999), suggesting a strong interrelatedness between ASD and ADHD, as a consequence of symptom overlap, of commonalities in etiology or both. In addition to these findings, one study suggested that the number of autism symptoms in children with OCD is related to comorbid ADHD (Ivarsson and Melin 2008).

These findings suggest that autism, ADHD, and OCD are interrelated. However, little research has been conducted to further elucidate the nature of this relationship at a symptom level. Insight in the association of co-morbid ADHD and autism symptoms might impact both etiological and treatment studies. Therefore, we aimed at extending current knowledge on symptom overlap between OC, ADHD and autism symptoms in adult OCD patients by investigating (1) whether autism and ADHD symptoms occur more frequently in OCD patients than in controls; (2) to what extent ADHD co-morbidity and autism symptom profiles in OCD predict symptom severity, and (3) to what extent ADHD and autism symptoms are related to specific OC symptom dimensions.

Based on previous investigations, we expected to find (1) elevated rates of autism and ADHD symptoms in OCD patients compared with healthy controls, (2) autism and ADHD symptoms predicting increased OC symptom severity. With respect to autism symptom characteristics in OCD, we expected (3) to replicate previous findings by our group of lack of social skills in the OCD group (Cath et al. 2008). Since perfectionism is found to be strongly associated with OCD (Libby et al. 2004), we expected (4) the OCD group to show increased attention to detail scale scores on the autism questionnaire. The attention to detail scores are expected to be highly correlated with the Y-BOCS scores. We expected (5) the hoarding symptom dimension of OCD to be most strongly associated with autism symptoms, since hoarding behavior is commonly reported in ASD (McDougle et al. 1995), and since reversely, hoarding in OCD is associated with social impairment and interpersonal problems (Grisham et al. 2008).

\section{Methods}

This study encompassed 109 OCD patients recruited from the anxiety outpatient mental health care services of GGZinGeest, Amsterdam (39\% males, mean age 37.5 years, SD 
10.0), and 87 healthy controls (53\% males, mean age 37.6 years, SD 13.6) who were recruited via the snowball method. Colleagues at the anxiety outpatient clinic were asked to recruit subjects whom they were acquainted with but were not close friends (to reduce risk of bias in this group). The control group was added to investigate whether OCD patients exhibit increased ADHD and autism symptoms levels compared with healthy controls. The study was approved by the Medical Ethics Committee of the Dutch Mental Health Care Institutes. All study subjects were included after written informed consent had been given. All patients were administered the Structured Clinical Interview on DSM-IV diagnoses (SCID-I) to establish inclusion and exclusion criteria (First 1996). Control subjects were administered the screening questions of the SCID-I. When responses on the screening questions were negative, they were invited for the study. Fulfilling criteria for a DSM IV OCD diagnosis was the inclusion criterion for the OCD patient group. OCD patients with psychosis, severe major depression, substance dependence, mental deficiency or inability to read/speak Dutch were excluded. The use of psychiatric medications in the OCD group included: $51.4 \%$ antidepressants, $6.4 \%$ antipsychotics, and $16.5 \%$ benzodiazepines.

\section{Measures}

\section{Autism Symptoms}

To rate autism symptoms all subjects completed a Dutch translation of the Autism-Spectrum Quotient (AQ; BaronCohen et al. 2001; Hoekstra et al. 2008). The AQ entails a 50 item self-administered instrument specifically developed for adults with normal intelligence with scores on each item between 1 (I fully agree) and 4 (I fully disagree). Either dichotomous ratings (range 0-50, with a cut-off $\geq 32$ suggestive of an autism spectrum disorder) or continuous (quantitative) ratings (range 50-200) can be computed from these scores. The dichotomous score as originally proposed by Baron-Cohen et al. (2001) is calculated by dichotomizing the item scores that range from 1-4 to 1-2 scores (or in case of reversed item formulations to 3-4 scores) a score of 1 is assigned indicating a positive score for ASD, and to 3-4 scores (or in case of reversed item formulation to 1-2 scores) a score of 0 is assigned indicating no ASD. The AQ has been validated in The Netherlands (Hoekstra et al. 2008) and contains five subscales with each 10 items: social skills (e.g. I find it hard to make new friends), attention switching (e.g. I frequently get so strongly absorbed in one thing that I lose sight of other things), attention to detail (e.g. I usually notice car number plates or similar strings of information), communication problems (e.g. other people frequently tell me that what
I've said is impolite, even though I think it is polite), and (lack of) imagination (e.g. when I'm reading a story, I find it difficult to work out the characters' intentions). Moderate to high internal consistency has been found in each of the five subscales (Hoekstra et al. 2008; Cronbach's $\alpha$ between .5 and .8)

\section{Attention Deficit and Hyperactivity (ADHD) Symptoms}

To measure attention deficit and hyperactivity (ADHD) symptoms, an interview was used to establish probable diagnoses of the various subtypes of ADHD according to DSM-IV criteria (inattentive type, hyperactive/impulsive or the combination), encompassing a Dutch version of the ADHD interview of DuPaul et al. (1998), translated, validated and adapted for the Dutch situation (Kooij et al. 2005). Individuals were asked separately about symptoms in the past and present (yes $=0 ;$ no $=1$ ), and two subscales are identified: a 9-item inattentive symptoms subscale and a 9-item hyperactive-impulsive symptoms subscale. A score of $\geq 6$ on the hyperactive/impulsive subscale indicates ADHD predominantly hyperactive/ impulsive type, while a score of $\geq 6$ on the inattentive subscale indicates ADHD predominantly inattentive type, and a score of $\geq 5$ on both subscales indicates a combined type of ADHD (Kooij et al. 2005). Since we regarded reports on present symptoms of ADHD to be more reliable than reports on past symptoms, for this study only the scores on present symptoms were used. Only present symptoms that were reported as being present since childhood were used.

\section{OCD Symptoms}

To asses OC symptoms, an 80-item self-report version of the Yale-Brown obsessive-compulsive symptom checklist (Y-BOCS) was used (Goodman et al. 1989a, b). To reduce the dataset in this small sample, item scores of the Y-BOCS symptom checklist were summarized into 4 symptom dimensions according to the 4 factor structure found by Leckman and colleagues and repeatedly replicated in large OCD samples (Leckman et al. 1997; Summerfeldt et al. 1999). These symptom dimensions entail: an aggression/checking factor, a symmetry/ordering factor, a contamination/washing factor and a hoarding factor. Further, the interview version of the 10 item Y-BOCS severity scale (scoring range 0-40) was used (Woody et al. 1995). During the interview, symptoms that patients reported in the Y-BOCS self-report were discussed, and only items that were assessed as clinically reliable were retained. In a recent study, the $\alpha$ of the Y-BOCD severity was .8 , and the $\alpha$ of the Y-BOCS symptom checklist .72 (Anholt et al. 2009). 
Statistical Analyses

To investigate interrelationships between the scales, and internal consistency of the measures Pearson's correlations and Cronbach's alpha's were calculated, using patient scale scores.

To calculate differences in demographic characteristics and clinical measures between OCD patients and normal controls, univariate variance analyses were used. Education level was operationalised in 3 levels: low (primary school and/or secondary low education; middle (primary school + secondary middle professional education); and high (either higher secondary education or high school and/ or high professional education or university). Since statistical significant differences were found in education levels between OCD subjects and normal controls $(p<.01)$, education was used as a covariate in the subsequent analyses. $\mathrm{X}^{2}$ analyses were used to analyze the dichotomous variables. AQ data were analyzed using both dichotomous scores as suggested by Baron-Cohen et al. to calculate cutoff scores of ASD (Baron-Cohen et al. 2001), and quantitative scores to compare quantitative measures of autism, ADHD and OC symptoms. Subsequent analyses concerned the OCD patient group only. OCD patients with versus without comorbid ADHD were compared on autism and OC symptom severity scores using ANOVAs. Finally, to assess which autism symptom subscales, ADHD subscales, or demographic variables predict OC symptom severity in OCD, stepwise linear regression analyses were run, using demographic variables and clinical subscales as independent variables, and Y-BOCS severity scores as dependent variable. Considering the number of variables used (12 subscales), Bonferroni correction was applied, and $p \leq .008$ was considered significant.

\section{Results}

Cronbach's $\alpha$ 's of all scale scores were reasonable to high. Cronbach's $\alpha$ 's of the AQ ranged between .57 (lack of imagination subscale) and .79 (AQ total scale), Of the Y-BOCS severity scale, $\alpha$ 's ranged between: .88 (obsession severity) and .91 (total severity), and $\alpha$ values of the Y-BOCS symptom dimensions ranged between .69 (hoarding subscale) and .87 (contamination/washing subscale). Cronbach's $\alpha$ scores of the ADHD subscale scores ranged between .79 (hyperactivity/impulsivity) and .85 (combined ADHD).

\section{Correlations}

AQ total scores were significantly correlated with ADHD total scores $(r=.52, p<01)$ and Y-BOCS total severity scores $(r=.56, p<.01)$. ADHD total scores exhibited significant correlations with the Y-BOCS severity scores $(r=.39, p<.01)$, as well as with the OC symptom domains aggression/checking $(r=.27, p<.05)$, symmetry/ordering $(r=.29, p<.01)$, and contamination/washing $(r=.4, p<.01)$. There were no significant correlations between ADHD total scores and hoarding ( $r=.17$, NS).

Pearson correlations were calculated between subscales of the various clinical measures (Table 3 ). The AQ subscales social skills and attention switching were significantly correlated with the ADHD subscales inattention ( $r=.33 ; p<.01$, and $r=.41 ; p<.01$, respectively), and hyperactivity $(r=.21 ; p<.05$, and $r=.25 ; p<.01$, respectively) and with all OC symptom dimensions ( $r$ between .18 and $.45 ; p$ between .05 and .01 ). Contrary to our expectation, the attention to detail AQ subscale demonstrated low correlation with OCD symptoms and severity (Table 1).

\section{OCD Versus Controls (Table 2)}

In accordance with our hypothesis, OCD patients showed significantly higher AQ and ADHD scores than the controls, on total AQ and ADHD scores as well as on all subscale scores except for the AQ imagination subscale. In the OCD group, $4.6 \%$, scored above the cut-point (Baron-Cohen et al. 2001) suggestive of ASD, versus $1.2 \%$ of the normal controls $(p=$ n.s.). Further, $11.0 \%$ of the OCD patients fulfilled the criteria suggestive of ADHD inattentiveness, $3.7 \%$ fulfilled criteria suggestive of ADHD hyperactivity/ impulsivity, and $8.2 \%$ fulfilled criteria suggestive of ADHD combined type. In the control group, $2.8 \%$ of the persons showed scores suggestive of ADHD inattentiveness, and no control subjects showed scores suggestive of hyperactive or combined type of ADHD (Table 2).

OCD + ADHD Patients Compared with OCD Without ADHD Patients (Table 3)

Twenty-five OCD patients showed ADHD scores suggestive of any ADHD diagnosis (OCD + ADHD), compared to 84 ADHD-free OCD patients (OCD - ADHD). OCD + ADHD patients scored higher than OCD - ADHD on total scores of the AQ, and on all AQ subscales (including communication, social skills, attention switching and imagination subscales), except for the attention to detail subscale. No between-group differences were found on Y-BOCS symptom severity (Table 3).

Linear Regression Analyses (Table 4)

In accordance with our hypothesis, the attention switching and communication subscales of the $\mathrm{AQ}$, the ADHD 
Table 1 Correlations between subscales of AQ, ADHD, Y-BOCS severity, and Y-BOCS symptom dimensions in OCD patients (correlations $>.3$ are marked in red)

\begin{tabular}{|c|c|c|c|c|c|c|c|c|c|c|c|c|}
\hline & $\begin{array}{l}\text { AQ } \\
\text { Soc }\end{array}$ & $\begin{array}{l}\text { AQ } \\
\text { Att } S\end{array}$ & $\begin{array}{l}\text { AQ } \\
\text { Att D }\end{array}$ & $\begin{array}{l}\text { AQ } \\
\text { Comm }\end{array}$ & $\begin{array}{l}\text { AQ } \\
\text { Imag }\end{array}$ & $\begin{array}{l}\text { ADHD } \\
\text { Inatt }\end{array}$ & $\begin{array}{l}\text { ADHD } \\
\text { Hyper }\end{array}$ & $\begin{array}{l}\text { Y-BOCS } \\
\text { Severity }\end{array}$ & $\begin{array}{l}\text { Y-BOCS } \\
\text { Aggres }\end{array}$ & $\begin{array}{l}\text { Y-BOCS } \\
\text { Sym }\end{array}$ & $\begin{array}{l}\text { Y-BOCS } \\
\text { Cont }\end{array}$ & $\begin{array}{l}\text { Y-BOCS } \\
\text { Hoard }\end{array}$ \\
\hline AQ Soc & 1 & $.81 * *$ & $.47 * *$ & $.71 * *$ & $.59 * *$ & $.33 * *$ & $.21 *$ & .17 & $.34 * *$ & $.31 * *$ & $.33 * *$ & $.18^{*}$ \\
\hline AQ Att S & & 1 & $.51 * *$ & $.68 * *$ & $.51 * *$ & $.41 * *$ & $.25 * *$ & $.45^{* *}$ & $.44 * *$ & $.42 * *$ & $.45^{* *}$ & $.27 * *$ \\
\hline AQ Att D & & & 1 & $.4 * *$ & $.32 * *$ & $.24 * *$ & $.19 * *$ & $.27 * *$ & $.18^{*}$ & $.2^{*}$ & $.23 * *$ & .09 \\
\hline AQ Comm & & & & 1 & $.6 * *$ & $.3 * *$ & $.3 * *$ & .14 & $.17 *$ & $.18^{*}$ & $.2 * *$ & .1 \\
\hline AQ Imag & & & & & 1 & $.27 * *$ & .15 & -.02 & .11 & .12 & $.18^{*}$ & $.16^{*}$ \\
\hline ADHD Inatt & & & & & & 1 & $.53 * *$ & $.28 * *$ & $.32 * *$ & $.37 * *$ & $.42 * *$ & $.22 *$ \\
\hline ADHD 2 Hyper & & & & & & & 1 & $.26^{*}$ & $.31 * *$ & $.27 * *$ & $.34 * *$ & .09 \\
\hline Y-BOCS Severity & & & & & & & & 1 & $.35^{* *}$ & $.42 * *$ & $.39 * *$ & .16 \\
\hline Y-BOCS Aggres & & & & & & & & & 1 & $.68 * *$ & $.68 * *$ & $.37 * *$ \\
\hline Y-BOCS Sym & & & & & & & & & & 1 & $.56^{* *}$ & $.37 * *$ \\
\hline Y-BOCS Cont & & & & & & & & & & & 1 & $.35^{* *}$ \\
\hline Y-BOCS Hoard & & & & & & & & & & & & 1 \\
\hline
\end{tabular}

$A Q$ Soc social skills, $A Q$ Att $S$ attention switching, $A Q$ Att $D$ attention to detail, $A Q$ Comm communication, $A Q$ Imag imagination, $A D H D$ Inatt inattention, $A D H D$ Hyper hyperactivity, $Y$-BOCS Severity total severity scores, $Y$-BOCS Aggres aggression/checking factor, $Y$-BOCS Sym symmetry/ordering factor, Y-BOCS Cont contamination/washing factor, Y-BOCS Hoard hoarding factor

Table 2 Demographic characteristics and clinical measures of patients and controls

\begin{tabular}{|c|c|c|c|c|}
\hline & OCD $(n=109)$ & Controls $(n=87)$ & $F$ & $p$ \\
\hline Males (\%) & $39.4 \%$ & $52.9 \%$ & 3.5 & NS \\
\hline Females $(\%)$ & $60.6 \%$ & $47.1 \%$ & & \\
\hline \multirow[t]{3}{*}{ Education level } & Low $11 \%$ & Low $22.3 \%$ & 16.4 & $<.01$ \\
\hline & Middle $11 \%$ & Middle $28.2 \%$ & & \\
\hline & High $75.2 \%$ & High 49.4 & & \\
\hline Age & $37.5(10.0)$ & $37.6(13.6)$ & .004 & NS \\
\hline Age of onset & $17.0(8.2)$ & - & - & - \\
\hline AQ total score & $114.6(17.1)$ & $95.1(15.1)$ & 68.8 & $<.01$ \\
\hline AQ social skills & $21.4(6.7)$ & $17.6(4.4)$ & 43.3 & $<.001$ \\
\hline AQ attention switching & $25.1(7.6)$ & $19.3(4.3)$ & 82.6 & $<.001$ \\
\hline AQ attention to detail & $23.4(5.6)$ & $20.3(4.6)$ & 17.6 & $<.001$ \\
\hline AQ communication & $20.1(6.1)$ & $18.0(3.8)$ & 22.3 & $<.001$ \\
\hline AQ imagination & $21.0(4.6)$ & $19.87(4.6)$ & 7.2 & NS \\
\hline ADHD total score & $4.9(4.1)$ & $2.44(3.0)$ & 11.7 & $<.001$ \\
\hline Inattention & $2.7(2.4)$ & $1.5(2.1)$ & 4.2 & $<.01$ \\
\hline Hyperactivity/impulsivity & $2.2(2.3)$ & $.9(1.3)$ & 12.4 & $<.01$ \\
\hline
\end{tabular}

$A D H D$ attention-deficit/hyperactivity disorder, $O C D$ obsessive-compulsive disorder, $A Q$ autism questionnaire, $Y$ - $B O C S$ Yale-Brown obsessive compulsive scale

hyperactivity subscale, and gender, accounted for $33 \%$ of the variation in OC symptom severity. Female gender was a weak predictor of OC symptom severity. Further, the AQ subscales attention switching, the ADHD inattention subscale, and AQ communication problems were the most important predictors of OC symptom dimensions $\left(r^{2}=\right.$ $.2-.31, p<.001)$ with the exception of hoarding. The finding that autism symptoms were not predictive of hoarding symptoms was contrary to our hypothesis. Older age and ADHD inattentive symptoms were positive predictors of hoarding symptoms. Only for the OC symptom dimension aggression and checking, the ADHD hyperactivity subscale and level of attained education were significant beyond the AQ subscales of attention switching and communication problems. Interestingly, only for the OC symptom dimension of contamination and washing 
Table 3 Mean scores of autism, OC symptom dimensions, symptom severity and ADHD scale scores in OCD \pm ADHD patients

\begin{tabular}{|c|c|c|c|c|}
\hline & $\begin{array}{l}\mathrm{OCD}-\mathrm{ADHD}(n=84) \\
m(\mathrm{SD})\end{array}$ & $\begin{array}{l}\mathrm{OCD}+\mathrm{ADHD}(n=25) \\
m(\mathrm{SD})\end{array}$ & $F$ & $p$ \\
\hline AQ total score & $109.4(15.6)$ & $126.1(18.9)$ & 16.4 & $<.001$ \\
\hline AQ social skills & $21.0(4.4)$ & $25.4(6.5)$ & 12.4 & $<.01$ \\
\hline AQ attention switching & $24.6(5.3)$ & $29.2(6.4)$ & 10.9 & $<.01$ \\
\hline AQ attention to detail & $23.3(4.8)$ & $25.0(6.6)$ & 1.6 & NS \\
\hline AQ communication & $19.8(4.5)$ & $22.9(4.6)$ & 7.6 & $<.01$ \\
\hline AQ imagination & $20.6(4.3)$ & $23.5(5.3)$ & 6.4 & NS \\
\hline Y-BOCS severity & $19.4(8.8)$ & $21.9(7.8)$ & 1.3 & NS \\
\hline Y-BOCS aggression/checking & $3.8(3.3)$ & $7.5(5.0)$ & 14.6 & $<.001$ \\
\hline Y-BOCS symmetry/ordering & $1.9(2.3)$ & $3.6(3.6)$ & 6.3 & NS \\
\hline Y-BOCS contamination/washing & $1.8(2.5)$ & $4.1(3.3)$ & 10.8 & $<.01$ \\
\hline Y-BOCS hoarding & $.2(.5)$ & $.2(.6)$ & .01 & NS \\
\hline ADHD total score & $3.4(2.8)$ & $10.5(3.0)$ & 91.4 & $<.001$ \\
\hline Inattention & $1.9(1.7)$ & $5.6(2.4)$ & 59.8 & $<.001$ \\
\hline Hyperactivity/impulsivity & $1.5(1.6)$ & $5.0(2.4)$ & 55.8 & $<.001$ \\
\hline
\end{tabular}

$A D H D$ attention-deficit/hyperactivity disorder, $O C D$ obsessive-compulsive disorder, $A Q$ autism questionnaire

ADHD inattention was the strongest predictor, whereas for the other OC symptom dimensions (besides hoarding) the AQ subscale of attention switching was the strongest predictor (Table 4).

\section{Discussion}

This is the first study to have systematically investigated the interrelations between OC, ADHD, and autism symptoms in adult OCD patients. As expected, OCD patients presented with higher scores on ADHD and autism symptoms than normal controls. OCD + ADHD patients showed higher autism scores on most autism subscales than OCD - ADHD patients, although no between-group differences in OC symptom severity were found. These findings are in line with earlier findings in pediatric OCD, and suggest an interrelatedness of ADHD symptoms with autism symptoms in OCD (Ivarsson and Melin 2008).

A substantial relationship between ADHD (particularly the inattentive and combined subgroups) and autism symptoms has not only been found in clinical OCD samples (as in the present study) but also in ADHD clinical as well as population-based samples (Reiersen et al. 2007). Family research has indicated that autism traits, particularly in males with ADHD, might represent a familial trait that is partly shared with ADHD (Mulligan et al. 2009). Thus, although according to DSM IV classification, ADHD and autism diagnoses are considered to be mutually exclusive, a growing body of literature (including the present small scale study) indicates that this classification rule does not hold. Moreover, autism and ADHD symptoms seem to share commonalities with OCD dimensions as well.

The interrelatedness between ADHD and autism symptoms within our OCD sample might reflect symptom overlap across the co-morbid disorders. Particularly the AQ attention switching subscale was highly correlated with the inattention subdomain of ADHD. Inspection of the wording of the items revealed seemingly contradictory relations between AQ attention switching and ADHD inattention: whereas inattention is expressed by the difficulty to sustain attention on a given task (e.g. "easily distracted because of external stimuli"), attention switching problems are expressed by the difficulty to divert attention between tasks (e.g. "I frequently get so strongly absorbed in one thing that I lose sight of other things"). Although seemingly contradictory, ADHD patients have repeatedly been found to exhibit both difficulty in attention switching (related to hyperfocusedness) and attention problems (Cepeda et al. 2000; Oades and Christiansen 2008). In this study, AQ problems with attention switching and ADHD inattention proved to be the strongest predictor for OC symptom severity, and of all OC symptom dimensions except hoarding. Therefore, AQ attention switching problems and ADHD inattention might well be at two ends of the same underlying attention problems.

The social skills subscale of the AQ showed significant correlations with both ADHD scales and OC symptom dimensions. Deficits in social skills have been found, both in OCD (Cath et al. 2008) and in ADHD (Reiersen et al. 2007). Thus, apart from the potential artifacts introduced by symptom overlap in measurements between the co-morbid disorders, ASD, ADHD and OCD might share 
Table 4 Stepwise regression analyses to predict OC symptom severity in OCD

\begin{tabular}{|c|c|c|c|c|c|}
\hline Dependent variable & Variable & $R$ & Adjusted $R$ square & Beta & $F$ and $p$ \\
\hline \multirow[t]{10}{*}{ Y-BOCS severity } & AQ attention switching & .45 & .20 & .45 & $31.2, p<.001$ \\
\hline & AQ attention switching & .51 & .25 & .67 & $20.9, p<.001$ \\
\hline & AQ communication & & & -.32 & \\
\hline & AQ attention switching & .58 & .32 & .64 & $19.9, p<.001$ \\
\hline & AQ communication & & & -.40 & \\
\hline & ADHD hyperactivity & & & .3 & \\
\hline & AQ attention switching & 6 & .33 & .64 & $16.3, p<.001$ \\
\hline & AQ communication & & & -.40 & \\
\hline & ADHD hyperactivity & & & .28 & \\
\hline & Gender & & & .15 & \\
\hline \multirow[t]{10}{*}{ Aggression and checking } & AQ attention switching & .41 & .16 & .40 & $24.7, p<.001$ \\
\hline & AQ attention switching & .46 & .20 & .60 & $16.0, p<.001$ \\
\hline & AQ communication & & & -.28 & \\
\hline & AQ attention switching & .51 & .25 & .57 & $14.3, p<.001$ \\
\hline & AQ communication & & & -.34 & \\
\hline & ADHD hyperactivity & & & .25 & \\
\hline & AQ attention switching & .55 & .27 & .57 & $12.6, p<.001$ \\
\hline & AQ communication & & & -.30 & \\
\hline & ADHD hyperactivity & & & .27 & \\
\hline & Level of attained education & & & .19 & \\
\hline \multirow[t]{6}{*}{ Symmetry and ordering } & AQ attention switching & .42 & .17 & .42 & $25.9, p<.001$ \\
\hline & AQ attention switching & .47 & .20 & .33 & $16.8, p<.001$ \\
\hline & ADHD inattention & & & .23 & \\
\hline & AQ attention switching & .5 & .23 & .48 & $13.0, p<.001$ \\
\hline & ADHD inattention & & & .23 & \\
\hline & AQ communication & & & -.23 & \\
\hline \multirow[t]{6}{*}{ Contamination and washing } & ADHD inattention & .44 & .19 & .44 & $29.8, p<.001$ \\
\hline & ADHD inattention & .52 & .26 & .32 & $23.1, p<.001$ \\
\hline & AQ attention switching & & & .31 & \\
\hline & ADHD inattention & .57 & .31 & .33 & $19.4, p<.001$ \\
\hline & AQ attention switching & & & .52 & \\
\hline & AQ communication & & & -.31 & \\
\hline \multirow[t]{3}{*}{ Hoarding } & Age & .25 & .06 & .25 & $8.11, p<.01$ \\
\hline & Age & .32 & .09 & .24 & $7.14, p<.01$ \\
\hline & ADHD inattention & & & .21 & \\
\hline
\end{tabular}

substantial etiology. Therefore, it would be worthwhile to reconsider the DSM-IV exclusionary classification of these disorders. In support of this, recent comparative studies between children with ASD and ADHD indicated that ASD children show differences as well as similarities on executive function when compared to ADHD and OCD children. Executive function refers to those neuropsychological processes that involve planning, strategic organization of complex processes, flexible adaptation to novel stimuli, ability to shift mental sets, to inhibit irrelevant (motor) responses, and to engage in trial and error learning (Purcell et al. 1998). Although substantial overlap existed between
ASD and ADHD children, in general ASD children performed worse than ADHD children on tasks of planning and flexibility, whereas ADHD children were worse on motor inhibition tasks and spatial working memory tasks, (Sinzig et al. 2008). Interestingly, OCD patients (Greisberg and McKay 2003; van den Heuvel et al. 2006) and in some instances their first degree relatives show problems in both domains; planning deficits comparable to ASD (Delorme et al. 2007), and impaired motor inhibition on similar tasks as found in ADHD patients (Sinzig et al. 2008; Chamberlain et al. 2007). Thus, OCD patients and their relatives seem to share executive function problems, both with ADHD and 
ASD patients, strongly suggesting overlapping etiologies between the disorders. Therefore, to study common etiologies among OCD, ADHD and ASD patients, an endophenotypic family-based approach is warranted in which executive function associated with underlying genetic mechanisms is studied in OCD, ADHD and ASD in concert.

Our hypothesis that hoarding symptoms were related with autism symptoms was not confirmed. Although significant, correlations between hoarding and AQ scores were lower than between other OC dimensions and AQ. Further, autism scores did not predict hoarding symptoms, but hoarding seemed to be related with inattentiveness and with older age rather than with autism symptoms, which is in line with the literature. Problems with focused attention, coupled with problems in decision making, categorization, and planning, have frequently been described in hoarders (Saxena 2008). With respect to age, the frequency and recognition of hoarding behavior is substantially increased among persons over 40 years of age (Samuels et al. 2008). Thus, this co-morbidity pattern, i.e. the association between attention problems rather than autism symptoms and hoarding on the other hand, suggests that-in line with the literature-hoarding constitutes a phenotype that is in several (but not all) aspects discrete from the other OC symptom dimensions (Abramowitz et al. 2008; An et al. 2009). However, no definite conclusions can be drawn from this purely descriptive study, since the hoarding subscale entails two Y-BOCS symptoms only, which hampers the sensitivity to pick up real differences.

The finding that the AQ lack of social skills scale was associated with OC symptom severity was in line with previous findings (Cath et al. 2008). Lack of social skills in OCD might reflect subtle impairments in theory of mind related functioning, a core feature in autism patients. Interestingly, theory of mind dysfunction is related to dysfunctions in set shifting and planning skills in children with autism (Pellicano 2007), and-although not investigated-might also be present in OCD patients at the severe end of the spectrum.

Contrary to our expectation, the AQ attention to detail subscale exhibited low correlations with OCD, and did not turn out to be a significant predictor of OC symptoms or severity. Apparently, the AQ attention to detail subscale taps on a trait that differs from perfectionism, a trait known to be closely related to (some forms of) OCD (Myers et al. 2008). Although patients are known to show enhanced attention for details related to OCD-relevant topics, these data suggest that in general, rather than scanning stimuli with great care to detail, OCD patients suffer from attention problems.

Recent research on memory distrust in OCD has found that OCD patients exhibit lack of confidence in their own perception, attention and memory, without clear evidence for an objective memory deficit (Hermans et al. 2008, 2003; Radomsky et al. 2001; Radomsky and Rachman 1999). Moreover, the repetitive behaviors of OCD seem to weaken memory trust (Van den Hout and Kindt 2004). However, the cause of the initial low confidence of OCD patients in their own memory capacities is unclear. Our findings of inattention and attention switch problems in OCD patients raise an interesting possibility, i.e. that these problems may, at least partially, be responsible for the low confidence of OCD patients in their cognitive processes. Difficulties in the management of attention may serve as a common factor between OCD, ADHD and ASD symptoms and explain the pathological doubt/uncertainty that seems to characterize these disorders.

These findings have several implications for treatment. First, considering that inattention as found in this study is central to OC symptoms, one would expect that addition of Methylphenidate to treatment in OCD patients with comorbid ADHD symptoms will enhance treatment efficacy. Future treatment studies should be directed at exploring this. Further, several behavioral strategies such as task concentration training that have been found to improve attention in ADHD patients (O'Connell et al. 2008, 2006; White and Shah 2006) could be added to behavior therapy in OCD patients, and might improve treatment efficacy particularly in OCD + ADHD patients. Another implication for clinical practice involves the importance of assessment of autism symptoms in the treatment of OCD. Possibly, increased autism symptoms complicate treatment and limit treatment gains. Cognitive-behavior therapy with patients with increased autism symptoms might demand longer duration, and require adaptations of standard treatment protocols. Further research of the effect of autism symptoms on treatment response to OCD is needed to confirm these hypotheses.

Such ideas are further strengthened by the lack of social skills as reported by the OCD patients, a finding that implies that adding a social skills improvement module to standard cognitive behavior therapy for OCD might increase overall treatment efficacy in OCD, as was recently suggested by Cath and colleagues (Cath et al. 2008).

Limitations of the present study include its descriptive nature, which precludes causal conclusions. Further, each symptom domain was measured by a single screening instrument. The measurement of ASD symptoms through self report alone is particularly problematic. Patients with complaints in the domain of self-reflection might be less able to reflect on their behavior. Further research should be conducted using multiple measures of the symptom domains of OCD, ADHD, and autism. Moreover, we suggest the use of more elaborated hoarding scales in future 
research, to further study relationship between autism and hoarding. The relations between pathological doubt and attention difficulties also need future exploration.

In conclusion: OCD patients exhibit increased rates of ADHD, and autism symptoms Problems with attention switching seems to be a strong predictor of OC severity and OC symptom dimensions, and might explain the lack of confidence in ones' own cognitive processes that are typical for OCD. Future research with various assessment measures, using a family-based paradigm across the OCD, ADHD and ASD phenotype, might help us to better understand the complex relationships between OCD, ADHD and ASD.

Open Access This article is distributed under the terms of the Creative Commons Attribution Noncommercial License which permits any noncommercial use, distribution, and reproduction in any medium, provided the original author(s) and source are credited.

\section{References}

Abramowitz, J. S., Wheaton, M. G., \& Storch, E. A. (2008). The status of hoarding as a symptom of obsessive-compulsive disorder. Behaviour Research and Therapy, 46, 1026-1033.

American Psychiatric Association (1994). Diagnostic and statistical manual of mental disorders (4th ed). Washington, DC.

An, S. K., Mataix-Cols, D., Lawrence, N. S., Wooderson, S., Giampietro, V., Speckens, A., et al. (2009). To discard or not to discard: The neural basis of hoarding symptoms in obsessivecompulsive disorder. Molecular Psychiatry, 14, 318-331.

Angst, J., Gamma, A., Endrass, J., Goodwin, R., Ajdacic, V., Eich, D., et al. (2004). Obsessive-compulsive severity spectrum in the community: Prevalence, comorbidity, and course. European Archives of Psychiatry and Clinical Neuroscience, 254, 156164.

Anholt, G. E., van Oppen, P., Emmelkamp, P. M. G., Cath, D. C., Smit, J. H., \& van Balkom, A. J. L. M. (2009). Measuring obsessive-compulsive symptoms: Padua inventory-revised vs. Yale-Brown obsessive compulsive scale. Journal of Anxiety Disorders, 23, 830-835.

Baron-Cohen, S., Wheelwright, S., Skinner, R., Martin, J., \& Clubley, E. (2001). The autism-spectrum quotient (AQ): Evidence from Asperger syndrome/high-functioning autism, males and females, scientists and mathematicians. Journal of Autism and Developmental Disorders, 31, 5-17.

Bebbington, P. E. (1998). Epidemiology of obsessive-compulsive disorder. British Journal of Psychiatry, 173(Suppl. 35), 2-6.

Bejerot, S. (2007). An autistic dimension: A proposed subtype of obsessive-compulsive disorder. Autism, 11, 101-110.

Bejerot, S., Nylander, L., \& Lindström, E. (2001). Autistic traits in obsessive-compulsive disorder. Nordic Journal of Psychiatry, $55,169-176$

Cath, D. C., Ran, N., Smit, J. H., van Balkom, A. J., \& Comijs, H. C. (2008). Symptom overlap between autism spectrum disorder, generalized social anxiety disorder and obsessive-compulsive disorder in adults: A preliminary case-controlled study. Psychopathology, 41, 101-110.

Cepeda, N. J., Cepeda, M. L., \& Kramer, A. F. (2000). Task switching and attention deficit hyperactivity disorder. Journal of Abnormal Child Psychology, 28, 213-226.
Chamberlain, S. R., Fineberg, N. A., Menzies, L. A., Blackwell, A. D., Bullmore, E. T., Robbins, T. W., et al. (2007). Impaired cognitive flexibility and motor inhibition in unaffected firstdegree relatives of patients with obsessive-compulsive disorder. American Journal of Psychiatry, 164, 335-338.

Clark, T., Feehan, C., Tinline, C., \& Vostanis, P. (1999). Autistic symptoms in children with attention deficit-hyperactivity disorder. European Child and Adolescent Psychiatry, 8, 50-55.

Delorme, R., Goussé, V., Roy, I., Trandafir, A., Mathieu, F., MourenSiméoni, M. C., et al. (2007). Shared executive dysfunctions in unaffected relatives of patients with autism and obsessivecompulsive disorder. European Psychiatry, 22, 32-38.

DuPaul, G. J., Power, T. J., Anastopoulos, A. D., \& Reid, R. (1998). $A D H D$ rating scale-IV: Checklists, norms and clinical interpretation. New York, NY: Guilford Publications, Inc.

First, M. B. (1996). Structured clinical interview for DSM-IV axis I disorders. Washington, DC: American Psychiatric Press, Inc.

Geller, D., Biederman, J., Faraone, S. V., Frazier, J., Coffey, B. J., Kim, G., et al. (2000). Clinical correlates of obsessive compulsive disorder in children and adolescents referred to specialized and non-specialized clinical settings. Depression and Anxiety, $11,163-168$.

Goldstein, S., \& Schwebach, A. J. (2004). The comorbidity of pervasive developmental disorder and attention deficit hyperactivity disorder: Results of a retrospective chart review. Journal of Autism and Developmental Disorders, 34, 329-339.

Goodman, W. K., Price, L. H., Rasmussen, S. A., Mazure, C., Delgado, P., Heninger, G. R., et al. (1989a). The Yale-Brown obsessive compulsive scale: II validity. Archives of General Psychiatry, 46, 1012-1016.

Goodman, W. K., Price, L. H., Rasmussen, S. A., Mazure, C., Fleischmann, R., Hill, C. L., et al. (1989b). The Yale-Brown obsessive-compulsive scale I: Development, use, and reliability. Archives of General Psychiatry, 46, 1006-1011.

Greisberg, S., \& McKay, D. (2003). Neuropsychology of obsessivecompulsive disorder: A review and treatment implications. Clinical Psychology Review, 23, 95-117.

Grisham, J. R., Steketee, G., \& Frost, R. O. (2008). Interpersonal problems and emotional intelligence in compulsive hoarding. Depression and Anxiety, 25, E63-E71.

Hanna, G. L. (1995). Demographic and clinical features of obsessivecompulsive disorder in children and adolescents. Journal of the American Academy of Child and Adolescent Psychiatry, 34, 19-27.

Hermans, D., Engelen, U., Grouwels, L., Joos, E., Lemmens, J., \& Pieters, G. (2008). Cognitive confidence in obsessive-compulsive disorder: Distrusting perception, attention and memory. Behaviour Research and Therapy, 46, 98-113.

Hermans, D., Martens, K., De Cort, K., Pieters, G., \& Eelen, P. (2003). Reality monitoring and metacognitive beliefs related to cognitive confidence in obsessive-compulsive disorder. Behaviour Research and Therapy, 41, 383-401.

Hoekstra, R. A., Bartels, M., Cath, D. C., \& Boomsma, D. I. (2008). Factor structure, reliability and criterion validity of the autismspectrum quotient (AQ): A study in Dutch population and patient groups. Journal of Autism and Developmental Disorders, 38 , $1555-1566$.

Hollander, E., Kwon, J. H., Stein, D. J., Broatch, J., Rowland, C. T., \& Himelein, C. A. (1996). Obsessive compulsive spectrum disorders: Overview and quality of life issues. Journal of Clinical Psychiatry, 57(suppl 8), 3-6.

Hollander, E., \& Wong, C. M. (1995). Obsessive compulsive spectrum disorders. Journal of Clinical Psychiatry, 46(Suppl. 4), 3-6.

Ivarsson, T., \& Melin, K. (2008). Autism spectrum traits in children and adolescents with obsessive-compulsive disorder (OCD). Journal of Anxiety Disorders, 22, 969-978. 
Kooij, J. J., Buitelaar, J. K., van den Oord, E. J., Furer, J. W., Rijnders, C. A., \& Hodiamont, P. P. (2005). Internal and external validity of attention-deficit hyperactivity disorder in a population-based sample of adults. Psychological Medicine, 35, 817827.

Leckman, J. F., Grice, D. E., Boardman, J., Zhang, H., Vitale, A., Bondi, C., et al. (1997). Symptoms of obsessive-compulsive disorder. American Journal of Psychiatry, 154, 911-917.

Leyfer, O. T., Folstein, S. E., Bacalman, S., Davis, N. O., Dinh, E., Morgan, J., et al. (2006). Comorbid psychiatric disorders in children with autism: Interview development and rates of disorders. Journal of Autism and Developmental Disorders, 36, 849-861.

Libby, S., Reynolds, S., Derisley, J., \& Clark, S. (2004). Cognitive appraisals in young people with obsessive-compulsive disorder. Journal of Child Psychology and Psychiatry and Allied Disciplines, 45, 1076-1084.

Maina, G., Albert, U., Salvi, V., Pessina, E., \& Bogetto, F. (2008). Early-onset obsessive-compulsive disorder and personality disorders in adulthood. Psychiatry Research, 158, 217-225.

March, J. S., \& Leonard, H. L. (1996). Obsessive-compulsive disorder in children and adolescents: A review of the past 10 years. Journal of the American Academy of Child and Adolescent Psychiatry, 35, 1265-1273.

McDougle, C. J., Kresch, L. E., Goodman, W. K., Naylor, S. T., Volkmar, F. R., Cohen, D. J., et al. (1995). A case-controlled study of repetitive thoughts and behavior in adults with autistic disorder and obsessive-compulsive disorder. American Journal of Psychiatry, 152, 772-777.

Mulligan, A., Anney, R. J., O’Regan, M., Chen, W., Butler, L., Fitzgerald, M., et al. (2009). Autism symptoms in attention deficit/hyperactivity disorder: A familial trait which correlates with conduct, oppositional defiant, language and motor disorders. Journal of Autism and Developmental Disorders, 39, 210-211.

Murray, C. J., \& Lopez, A. D. (1996). The global burden of disease. Boston, MA: Harvard University Press.

Myers, S. G., Fisher, P. L., \& Wells, A. (2008). Belief domains of the obsessive beliefs questionnaire-44 (OBQ-44) and their specific relationship with obsessive-compulsive symptoms. Journal of Anxiety Disorders, 22, 475-484.

Myers, J. K., Weissman, M. M., Tischler, G. L., Holzer, C. E., Leaf, P. J., Orvaschel, H., et al. (1984). Six-month prevalence of psychiatric disorders in three communities. Archives of General Psychiatry, 41, 959-970.

O'Connell, R. G., Bellgrove, M. A., Dockree, P. M., Lau, A., Fitzgerald, M., \& Robertson, I. H. (2008). Self-alert training: Volitional modulation of autonomic arousal improves sustained attention. Neuropsychologia, 46, 1379-1390.

O'Connell, R. G., Bellgrove, M. A., Dockree, P. M., \& Robertson, I. H. (2006). Cognitive remediation in ADHD: Effects of periodic non-contingent alerts on sustained attention to response. Neuropsychological Rehabilitation, 16, 653-665.

Oades, R. D., \& Christiansen, H. (2008). Cognitive switching processes in young people with attention-deficit/hyperactivity disorder. Archives of Clinical Neuropsychology, 23, 21-32.

Palumbo, D., Maughan, A., \& Kurlan, R. (1997). Hypothesis III. Tourtte syndrome is only one of several causes to a development basal ganglia syndromes. Archives of Neurology, 54, 475-481.

Pellicano, E. (2007). Links between theory of mind and executive function in young children with autism: Clues to developmental primacy. Developmental Psychology, 43, 974-990.

Purcell, R., Maruff, P., Kyrios, M., \& Pantelis, C. (1998). Cognitive deficits in obsessive-compulsive disorder on tests of frontalstriatal function. Biological Psychiatry, 43, 348-357.
Radomsky, A. S., \& Rachman, S. (1999). Memory bias in obsessivecompulsive disorder (OCD). Behaviour Research and Therapy, 37, 605-618.

Radomsky, A. S., Rachman, S., \& Hammond, D. (2001). Memory bias, confidence and responsibility in compulsive checking. Behaviour Research and Therapy, 39, 813-822.

Reiersen, A. M., Constantino, J. N., Volk, H. E., \& Todd, R. D. (2007). Autistic traits in a population-based ADHD twin sample. The Journal of Child Psychology and Psychiatry, 48, 464-472.

Russell, A. J., Mataix-Cols, D., Anson, M., \& Murphy, D. G. (2005). Obsessions and compulsions in Asperger syndrome and highfunctioning autism. British Journal of Psychiatry, 186, 525-528.

Samuels, J. F., Bienvenu, O. J., Grados, M. A., Cullen, B., Riddle, M. A., Liang, K. Y., et al. (2008). Prevalence and correlates of hoarding behavior in a community-based sample. Behaviour Research and Therapy, 46, 836-844.

Saxena, S. (2008). Recent advances in compulsive hoarding. Current Psychiatry Reports, 10, 297-303.

Sinzig, J., Morsch, D., Bruning, N., Schmidt, M. H., \& Lehmkuhl, G. (2008). Inhibition, flexibility, working memory and planning in autism spectrum disorders with and without comorbid ADHDsymptoms. Child and Adolescent Psychiatry and Mental Health, 2,4 .

Stein, M. B., Ford, D. R., Anderson, G., \& Walker, J. R. (1997). Obsessive-compulsive disorder in the community: An epidemiological survey with clinical reappraisal. American Journal of Psychiatry, 154, 1120-1126.

Sturm, H., Fernell, E., \& Gillberg, C. (2004). Autism spectrum disorders in children with normal intellectual levels: Associated impairments and subgroups. Developmental Medicine and Child Neurology, 46, 444-447.

Summerfeldt, L. J., Richter, M. A., Antony, M. M., \& Swinson, R. P. (1999). Symptom structure in obsessive-compulsive disorder: A confirmatory factor-analytic study. Behaviour Research and Therapy, 37, 297-311.

van den Heuvel, O. A., Veltman, D. J., Groenewegen, H. J., Cath, D. C., van Balkom, A. J. L. M., van Hartskamp, J., et al. (2006). Frontal-striatal dysfunction during planning in obsessive-compulsive disorder. Archives of General Psychiatry, 62, 301-309.

van den Hout, M., \& Kindt, M. (2004). Obsessive-compulsive disorder and the paradoxical effects of perseverative behaviour on experienced uncertainty. Journal of Behavior Therapy and Experimental Psychiatry, 35, 165-181.

Weissman, M. M., Bland, R. C., Canino, G. J., Greenwald, S., Hwu, H. G., Lee, C. K., et al. (1994). The cross national epidemiology of obsessive compulsive disorder. The Cross National Collaborative Group. Journal of Clinical Psychiatry, 55(suppl), 5-10.

White, H. A., \& Shah, P. (2006). Training attention-switching ability in adults with ADHD. Journal of Attention Disorders, 10, 44-53.

Woody, S. R., Steketee, G., \& Chambless, D. L. (1995). Reliability and validity of the Yale-Brown obsessive-compulsive scale. Behaviour Research and Therapy, 33, 597-605.

Yoshida, Y., \& Uchiyama, T. (2004). The clinical necessity for assessing attention deficit/hyperactivity disorder (AD/HD) symptoms in children with high functioning pervasive developmental disorder (PDD). European Child and Adolescent Psychiatry, 13, 307-314.

Zandt, F., Prior, M., \& Kyrios, M. (2007). Repetitive behaviour in children with high functioning autism and obsessive compulsive disorder. Journal of Autism and Developmental Disorders, 37, 251-265. 